\title{
center $\theta$
}

No. $2003-129$

\section{THE P-VALUE FOR COST SHARING IN MINIMUM COST SPANNING TREE SITUATIONS}

\author{
By R. Branzei, S. Moretti, H.W. Norde, S.H. Tijs
}

December 2003

ISSN 0924-7815

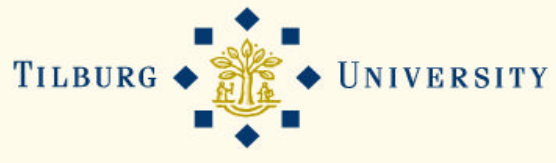




\title{
The $P$-value for cost sharing in minimum cost spanning tree situations ${ }^{1}$
}

\author{
Rodica Branzei ${ }^{2}$, Stefano Moretti ${ }^{3}$, Henk Norde ${ }^{4}$ and Stef Tijs ${ }^{5}$
}

August 1, 2003

Abstract: The aim of this paper is to introduce and axiomatically characterize the $P$-value as a rule to solve the cost sharing problem in minimum cost spanning tree (mcst) situations. The $P$-value is related to the Kruskal algorithm for finding an mcst. Moreover, the $P$-value leads to a core allocation of the corresponding mest game, and when applied also to the mcst subsituations it delivers a population monotonic allocation scheme. A conewise positive linearity property is one of the basic ingredients of an axiomatic characterization of the $P$-value.

Key-words: Cost sharing, minimum cost spanning tree games, value, population monotonic allocation schemes.

\section{Introduction}

Since the basic paper of Bird (1976) much attention has been paid to the problem of sharing costs in situations where agents have to be connected with a source as cheap as possible, and where connections between users and between users and the source can be shared among users if they cooperate. Let us refer to the dissertations of Aarts (1994) and Feltkamp (1995), and to the papers of Granot and Huberman (1981), Feltkamp et al. (1994), and Kar (2002). In the papers of Dutta and Kar (2002), Kent and Skorin-Kapov

\footnotetext{
${ }^{1}$ This paper is written to honour Guillermo Owen on the occasion of his $65^{\text {th }}$ birthday.

${ }^{2}$ Faculty of Computer Science, "Alexandru Ioan Cuza" University, Iasi, Romania.

${ }^{3}$ Department of Mathematics, University of Genova and Department of Environmental Epidemiology, National Cancer Research Institute of Genova, Italy.

${ }^{4}$ CentER and Department of Econometrics and Operations Research, Tilburg University, The Netherlands.

${ }^{5}$ CentER and Department of Econometrics and Operations Research, Tilburg University, The Netherlands and Department of Mathematics, University of Genova, Italy.
} 
(1996), Moretti et al. (2002), and Norde et al. (2001), the existence of cost monotonic and population monotonic allocation rules (Sprumont (1990)) is central.

The $P$-value introduced in Section 3 , has been arisen from our interest in monotonic allocation schemes too (Tijs et al. (2003)). Our introduction of the $P$-value is a two-step procedure. First, we define this value on cones of mest situations with the same ordering pattern of the edges with respect to costs. Then, we prove that we can patch these $P$-values together to the whole cone of mest situations. It turns out that our $P$-value equals the Equal Remaining Obligations (ERO) rule suggested by Jos Potters (which explains the name of our rule) and which is studied first in Feltkamp et al. (1994). Furthermore, our $P$-value turns out to be the average of the population monotonic allocation rules introduced in Norde et al. (2001). In Section 4 we give an axiomatic characterization of the $P$-value, where the cone-wise positive linearity of $P$ is a fundamental property and where the decomposition of an mest situation into simple mest situations (cf. Kuipers (1993), Norde et al. (2001)) plays a role. In Section 5, which concludes the paper, the related $\Pi$-value for minimum spanning tree games is introduced and it is shown that the $\Pi$-value is a population monotonic allocation rule.

\section{Preliminaries and notations}

First, we recall some definitions from graph theory which are used in this paper. An (undirected) graph is a pair $\langle V, E\rangle$, where $V$ is a set of vertices or nodes and $E$ is a set of edges $e$ of the form $\{i, j\}$ with $i, j \in V, i \neq j$. The complete graph on a set $V$ of vertices is the graph $\left\langle V, E_{V}\right\rangle$, where $E_{V}=\{\{i, j\} \mid i, j \in V$ and $i \neq j\}$. A path between $i$ and $j$ in a graph $<V, E>$ is a sequence of nodes $i=i_{0}, i_{1}, \ldots, i_{k}=j, k \geq 1$, such that $\left\{i_{s}, i_{s+1}\right\} \in E$ for each $s \in\{0, \ldots, k-1\}$. A cycle in $\langle V, E\rangle$ is a path from $i$ to $i$ for some $i \in V$. Two nodes $i, j \in V$ are connected in $(V, E)$ if $i=j$ or if there exists a path between $i$ and $j$ in $E$.

Now, we consider minimum cost spanning tree (mcst) situations. In an mcst situation a set $N=\{1, \ldots, n\}$ of agents is involved willing to be connected as cheap as possible to a source (i.e. a supplier of a service) denoted by 0 . In the sequel we use the notation $N^{\prime}=N \cup\{0\}$. An mcst situation can be represented by a tuple $<N^{\prime}, E_{N^{\prime}}, w>$, where $<N^{\prime}, E_{N^{\prime}}>$ is the complete graph on the set $N^{\prime}$ of nodes or vertices, and $w: E_{N^{\prime}} \rightarrow R_{+}$is a map which assigns to each edge $e \in E_{N^{\prime}}$ a nonnegative number $w(e)$ representing the weight or cost of edge $e$. We call $w$ a weight function. If $w(e) \in\{0,1\}$ for every $e \in E_{N^{\prime}}$, the weight function $w$ is called a simple weight function, and 
we refer then to $\left\langle N^{\prime}, E_{N^{\prime}}, w>\right.$ as a simple mcst situation.

Since in our paper the graph of possible edges is always the complete graph, we simply denote an mcst situation with set of users $N$, source 0 , and weight function $w$ by $\left\langle N^{\prime}, w\right\rangle$. Often we identify an mcst situation $\left.<N^{\prime}, w\right\rangle$ with the corresponding weight function $w$. We denote by $\mathcal{W}^{N^{\prime}}$ the set of all mcst situations $\left\langle N^{\prime}, w\right\rangle$ (or $w$ ) with node set $N^{\prime}$. For each $S \subseteq N$, one can consider the mcst subsituation $<S^{\prime}, w_{\mid S^{\prime}}>$, where $S^{\prime}=S \cup\{0\}$ and $w_{\mid S^{\prime}}: E_{S^{\prime}} \rightarrow \mathbb{R}_{+}$is the restriction of the weight function $w$ to $E_{S^{\prime}} \subseteq E_{N^{\prime}}$, i.e. $w_{\mid S^{\prime}}(e)=w(e)$ for each $e \in E_{S^{\prime}}$.

Let $\left\langle N^{\prime}, w\right\rangle$ be an mcst situation. Two nodes $i$ and $j$ are called $\left(w, N^{\prime}\right)$ connected if $i=j$ or if there exists a sequence of nodes $i=i_{0}, \ldots, i_{k}=j$ in $N^{\prime}, k \geq 1$, with $w\left(\left\{i_{s}, i_{s+1}\right\}\right)=0$ for every $s \in\{0, \ldots, k-1\}$. A $\left(w, N^{\prime}\right)$ component of $N^{\prime}$ is a maximal subset of $N^{\prime}$ with the property that any two nodes in this subset are $\left(w, N^{\prime}\right)$-connected. We denote by $C_{i}(w)$ the $\left(w, N^{\prime}\right)$-component to which $i$ belongs and by $\mathcal{C}(w)$ the set of all the $\left(w, N^{\prime}\right)$ components of $N^{\prime}$. Clearly, the collection of $\left(w, N^{\prime}\right)$-components forms a partition of $N^{\prime}$.

We define the set $\Sigma_{E_{N^{\prime}}}$ of linear orders on $E_{N^{\prime}}$ as the set of all bijections $\sigma:\left\{1, \ldots,\left|E_{N^{\prime}}\right|\right\} \rightarrow E_{N^{\prime}}$, where $\left|E_{N^{\prime}}\right|$ is the cardinality of the set $E_{N^{\prime}}$. For each mcst situation $\left\langle N^{\prime}, w>\right.$ there exists at least one linear order $\sigma \in \Sigma_{E_{N^{\prime}}}$ such that $w(\sigma(1)) \leq w(\sigma(2)) \leq \ldots \leq w\left(\sigma\left(\left|E_{N^{\prime}}\right|\right)\right)$. We denote by $w^{\sigma}$ the column vector $\left(w(\sigma(1)), w(\sigma(2)), \ldots, w\left(\sigma\left(\left|E_{N^{\prime}}\right|\right)\right)\right)^{t}$.

For any $\sigma \in \Sigma_{E_{N^{\prime}}}$ we define the set

$$
K^{\sigma}=\left\{w \in \mathbb{R}_{+}^{E_{N^{\prime}}} \mid w(\sigma(1)) \leq w(\sigma(2)) \leq \ldots \leq w\left(\sigma\left(\left|E_{N^{\prime}}\right|\right)\right)\right\} .
$$

The set $K^{\sigma}$ is a cone in $\mathbb{R}_{+}^{E_{N^{\prime}}}$, which we call the Kruskal cone with respect to $\sigma$. One can easily see that $\bigcup_{\sigma \in \Sigma_{E_{N^{\prime}}}} K^{\sigma}=\mathbb{R}_{+}^{E_{N^{\prime}}}$. For each $\sigma \in \Sigma_{E_{N^{\prime}}}$ the cone $K^{\sigma}$ is a simplicial cone with generators $e^{\sigma, k} \in K^{\sigma}, k \in\left\{1,2, \ldots,\left|E_{N^{\prime}}\right|\right\}$, where

$$
\begin{gathered}
e^{\sigma, k}(\sigma(1))=e^{\sigma, k}(\sigma(2))=\ldots=e^{\sigma, k}(\sigma(k-1))=0 \\
\text { and } \\
e^{\sigma, k}(\sigma(k))=e^{\sigma, k}(\sigma(k+1))=\ldots=e^{\sigma, k}\left(\sigma\left(\left|E_{N^{\prime}}\right|\right)\right)=1
\end{gathered}
$$

[Note that $e^{\sigma, 1}(\sigma(k))=1$ for all $\left.k \in\left\{1,2, \ldots,\left|E_{N^{\prime}}\right|\right\}\right]$.

This implies that each $w \in K^{\sigma}$ can be written in a unique way as nonnegative linear combination of these generators. To be more concrete, for $w \in K^{\sigma}$ we have

$$
w=w(\sigma(1)) e^{\sigma, 1}+\sum_{k=2}^{\left|E_{N^{\prime}}\right|}(w(\sigma(k))-w(\sigma(k-1))) e^{\sigma, k} .
$$


Clearly, we can also write $\mathcal{W}^{N^{\prime}}=\bigcup_{\sigma \in \Sigma_{E_{N^{\prime}}}} K^{\sigma}$, if we identify an mcst situation $\left\langle N^{\prime}, w>\right.$ with $w$.

Any mcst situation gives rise to two problems: the construction of a network $\Gamma \subseteq E_{N^{\prime}}$ of minimal cost connecting all users to the source, and a cost sharing problem of distributing this cost among users in a fair way. The cost of a network $\Gamma$ is $w(\Gamma)=\sum_{e \in \Gamma} w(e)$. A network $\Gamma$ is a spanning network on $S^{\prime} \subseteq N^{\prime}$ if for every $e \in \Gamma$ we have $e \in E_{S^{\prime}}$ and for every $i \in S$ there is a path in $\Gamma$ from $i$ to the source. The cost of a minimum (cost) spanning network $\Gamma$ on $N^{\prime}$ in a simple mest situation equals $|\mathcal{C}(w)|-1$ (see Lemma 2 in Norde et al. (2001)). To construct a minimum cost spanning network $\Gamma$ on $N^{\prime}$ we use in this paper the Kruskal algorithm (Kruskal (1956)), where the edges are considered one by one according to non-decreasing cost, and an edge is either rejected, if it generates a cycle with the edges already constructed, or it is constructed, otherwise.

Let $\left\langle N^{\prime}, w\right\rangle$ be an mest situation. The minimum cost spanning tree game $\left(N, c_{w}\right)$ (or simply $c_{w}$ ), corresponding to $\left\langle N^{\prime}, w\right\rangle$, is defined by

$$
c_{w}(S)=\min \left\{w(\Gamma) \mid \Gamma \text { is a spanning network on } S^{\prime}\right\}
$$

for every $S \in 2^{N} \backslash\{\emptyset\}$, where $2^{N}$ stands for the power set of the player set $N$, with the convention that $c_{w}(\emptyset)=0$.

We denote by $\mathcal{M C S T}^{N}$ the class of all mcst games corresponding to mcst situations in $\mathcal{W}^{N^{\prime}}$. For each $\sigma \in \Sigma_{E_{N^{\prime}}}$, we denote by $\mathcal{G}^{\sigma}$ the set $\left\{c_{w} \mid w \in K^{\sigma}\right\}$ which is a cone. We can express $\mathcal{M C S \mathcal { T } ^ { N }}$ as the union of all cones $\mathcal{G}^{\sigma}$, i.e. $\mathcal{M C S T ^ { N }}=\bigcup_{\sigma \in \Sigma_{E_{N}}} \mathcal{G}^{\sigma}$, and we would like to point out that $\mathcal{M C S T}^{N}$ itself is not a cone if $|N| \geq 2$.

\section{The $P$-value}

Let $w \in \mathcal{W}^{N^{\prime}}$ and let $\sigma \in \Sigma_{E_{N^{\prime}}}$ be such that $w \in K^{\sigma}$. We can consider a sequence of precisely $\left|E_{N^{\prime}}\right|+1$ graphs $\left\langle N^{\prime}, F^{\sigma, 0}\right\rangle,\left\langle N^{\prime}, F^{\sigma, 1}\right\rangle, \ldots$, $<N^{\prime}, F^{\sigma,\left|E_{N^{\prime}}\right|}>$ such that $F^{\sigma, 0}=\emptyset, F^{\sigma, k}=F^{\sigma, k-1} \cup\{\sigma(k)\}$ for each $k \in\left\{1, \ldots,\left|E_{N^{\prime}}\right|\right\}$. Now, we define the connection vectors $b^{\sigma, k} \in \mathbb{R}^{N}$ for each $k \in\left\{0,1, \ldots,\left|E_{N^{\prime}}\right|\right\}$ as follows

$$
b_{i}^{\sigma, k}= \begin{cases}0 & \text { if } i \text { is connected to } 0 \text { in }<N^{\prime}, F^{\sigma, k}> \\ \frac{1}{n_{i}\left(F^{\sigma, k}\right)} & \text { otherwise }\end{cases}
$$

for each $i \in N$, where $n_{i}\left(F^{\sigma, k}\right)$ is the number of nodes in $N$ which are connected to $i$, directly or indirectly, via edges in $F^{\sigma, k}$. Note that $n_{i}\left(F^{\sigma, k}\right)=1$ 
if $i$ is disconnected from each other node in $\left\langle N^{\prime}, F^{\sigma, k}\right\rangle$. Note also that for each $\sigma \in \Sigma_{E_{N^{\prime}},}, b_{i}^{\sigma, 0}=1$ and $b_{i}^{\sigma,\left|E_{N^{\prime}}\right|}=0$, for each $i \in N$.

Example 1 Consider the mest situation $\left\langle N^{\prime}, w\right\rangle$ with $N^{\prime}=\{0,1,2,3\}$ and $w$ as depicted in Figure 1. Note that $w \in K^{\sigma}$, with $\sigma(1)=\{1,3\}$, $\sigma(2)=\{1,2\}, \sigma(3)=\{2,3\}, \sigma(4)=\{1,0\}, \sigma(5)=\{2,0\}, \sigma(6)=\{3,0\}$.

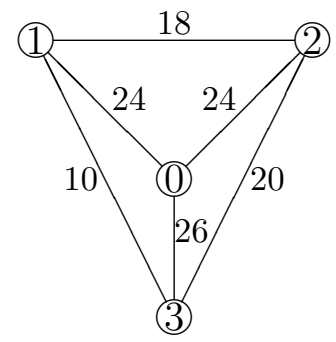

Figure 1: An mcst situation with three agents.

The sequence of seven graphs $\left\langle N^{\prime}, F^{\sigma, k}\right\rangle$ and the corresponding connection vectors $b^{\sigma, k}$ are shown in the following table

$$
\begin{array}{l|l}
<N^{\prime}, \emptyset> & b^{\sigma, 0}=(1,1,1)^{t} \\
<N^{\prime},\{\{1,3\}\}> & b^{\sigma, 1}=\left(\frac{1}{2}, 1, \frac{1}{2}\right)^{t} \\
<N^{\prime},\{\{1,3\},\{1,2\}\}> & b^{\sigma, 2}=\left(\frac{1}{3}, \frac{1}{3}, \frac{1}{3}\right)^{t} \\
<N^{\prime},\{\{1,3\},\{1,2\},\{2,3\}\}> & b^{\sigma, 3}=\left(\frac{1}{3}, \frac{1}{3}, \frac{1}{3}\right)^{t} \\
<N^{\prime},\{\{1,3\},\{1,2\},\{2,3\},\{1,0\}\}> & b^{\sigma, 4}=(0,0,0)^{t} \\
<N^{\prime},\{\{1,3\},\{1,2\},\{2,3\},\{1,0\},\{2,0\}\}> & b^{\sigma, 5}=(0,0,0)^{t} \\
<N^{\prime},\{\{1,3\},\{1,2\},\{2,3\},\{1,0\},\{2,0\},\{3,0\}\}> & b^{\sigma, 6}=(0,0,0)^{t}
\end{array}
$$

Remark 1 Let $\sigma \in \Sigma_{E_{N^{\prime}}}$. For each $k \in\left\{1, \ldots,\left|E_{N^{\prime}}\right|\right\}$, consider the simple mcst situation $e^{\sigma, k}$. Then, for $k>1$, each edge $e \in F^{\sigma, k-1}$ has cost $e^{\sigma, k}(e)=0$. Therefore, if $i$ and $j$ in $N^{\prime}$ are connected in $\left\langle N^{\prime}, F^{\sigma, k-1}\right\rangle$, then they are also in the same $\left(e^{\sigma, k}, N^{\prime}\right)$-component. Conversely, if $i$ and $j$ are in the same $\left(e^{\sigma, k}, N^{\prime}\right)$-component, then they are also connected in $\left\langle N^{\prime}, F^{\sigma, k-1}\right\rangle$ and as a consequence, by relation $(3), b_{i}^{\sigma, k-1}=b_{j}^{\sigma, k-1}$.

Definition 1 Let $\sigma \in \Sigma_{E_{N^{\prime}}}$. The contribution matrix w.r.t. $\sigma$ is the matrix $M^{\sigma} \in \mathbb{R}^{N \times E_{N^{\prime}}}$ where the rows correspond to the agents and the columns to the edges and such that the $k$-th column of $M^{\sigma}$ equals

$$
M^{\sigma} e^{k}=b^{\sigma, k-1}-b^{\sigma, k}
$$

for each $k \in\left\{1, \ldots,\left|E_{N^{\prime}}\right|\right\}$. [Here $e^{k}$ is a column vector such that $e_{i}^{k}=1$ if $i=k$ and $e_{i}^{k}=0$ for each $i \in\left\{1, \ldots,\left|E_{N^{\prime}}\right|\right\} \backslash\{k\}$.] 
Note that each column $M^{\sigma} e^{k}$ such that $\left(M^{\sigma} e^{k}\right)_{i} \neq 0$ for some $i \in N$ corresponds to the edge $\sigma(k)$ constructed at stage $k$ in Kruskal's algorithm. Note that the sum of the elements of such a column equals 1 . The interpretation of the contribution vector $M^{\sigma} e^{k}$ is that each entry $\left(M^{\sigma} e^{k}\right)_{i}, i \in N$, represents the fraction of the cost of edge $\sigma(k)$ paid by user $i$. On the other hand, the zero columns in $M^{\sigma}$ correspond to rejected edges in Kruskal's algorithm.

Another characteristic of the contribution matrix is that the sum of the elements in each row $i \in N$ is equal to 1 ,

$$
\sum_{k=1}^{\left|E_{N^{\prime}}\right|}\left(M^{\sigma} e^{k}\right)_{i}=\sum_{k=1}^{\left|E_{N^{\prime}}\right|}\left(b_{i}^{\sigma, k-1}-b_{i}^{\sigma, k}\right)=b_{i}^{\sigma, 0}-b_{i}^{\sigma,\left|E_{N^{\prime}}\right|}=1-0=1 .
$$

Definition 2 For each $\sigma \in \Sigma_{E_{N^{\prime}}}$, we define the $P^{\sigma}$-value as the map $P^{\sigma}$ : $K^{\sigma} \rightarrow \mathbb{R}^{N}$, where $P^{\sigma}(w)=M^{\sigma} w^{\sigma}$ for each mcst situation $w$ in the cone $K^{\sigma}$.

In order to define $P$ on $\mathcal{W}^{N^{\prime}}$ we need Proposition 1, which follows directly from the following lemma.

Lemma 1 Let $\sigma \in \Sigma_{E_{N^{\prime}}}, w \in K^{\sigma}$ and suppose that, for some $t \in\{1, \ldots$, $\left.\left|E_{N^{\prime}}\right|-1\right\}, w_{t}^{\sigma}=w_{t+1}^{\sigma}$. Then for the ordering $\sigma^{\prime} \in \Sigma_{E_{N^{\prime}}}$ such that $\sigma^{\prime}(i)=\sigma(i)$ for each $i \in\left\{1, \ldots,\left|E_{N^{\prime}}\right|\right\} \backslash\{t, t+1\}, \sigma^{\prime}(t)=\sigma(t+1)$ and $\sigma^{\prime}(t+1)=\sigma(t)$, we have that $w \in K^{\sigma^{\prime}}$ and $P^{\sigma}(w)=P^{\sigma^{\prime}}(w)$.

Proof It is obvious that $w \in K^{\sigma^{\prime}}$. Put $a=w_{t}^{\sigma}$. Note that $b^{\sigma, k}=b^{\sigma^{\prime}, k}$ for all $k \in\left\{1, \ldots,\left|E_{N^{\prime}}\right|\right\}$ with $k \neq t$. This implies that $w_{k}^{\sigma} M^{\sigma} e^{k}=w_{k}^{\sigma^{\prime}} M^{\sigma^{\prime}} e^{k}$ for all $k \in\left\{1, \ldots,\left|E_{N^{\prime}}\right|\right\}$ with $k \notin\{t, t+1\}$ and

$$
\begin{aligned}
& w_{t}^{\sigma^{\prime}} M^{\sigma^{\prime}} e^{t}+w_{t+1}^{\sigma^{\prime}} M^{\sigma^{\prime}} e^{t+1}= \\
& =a\left(b^{\sigma^{\prime}, t-1}-b^{\sigma^{\prime}, t}\right)+a\left(b^{\sigma^{\prime}, t}-b^{\sigma^{\prime}, t+1}\right)= \\
& =a\left(b^{\sigma^{\prime}, t-1}-b^{\sigma^{\prime}, t+1}\right)=a\left(b^{\sigma, t-1}-b^{\sigma, t+1}\right)= \\
& =a\left(b^{\sigma, t-1}-b^{\sigma, t}\right)+a\left(b^{\sigma, t}-b^{\sigma, t+1}\right)= \\
& =w_{t}^{\sigma} M^{\sigma} e^{t}+w_{t+1}^{\sigma} M^{\sigma} e^{t+1}
\end{aligned}
$$

So, $M^{\sigma} w^{\sigma}=M^{\sigma^{\prime}} w^{\sigma^{\prime}}$ or, equivalently, $P^{\sigma}(w)=P^{\sigma^{\prime}}(w)$.

Proposition 1 If $w \in K^{\sigma} \cap K^{\sigma^{\prime}}$ with $\sigma, \sigma^{\prime} \in \Sigma_{E_{N^{\prime}}}$, then $P^{\sigma}(w)=P^{\sigma^{\prime}}(w)$.

This proposition makes it possible to define the $P$-value on $\mathcal{W}^{N^{\prime}}$.

Definition 3 The $P$-value is the map $P: \mathcal{W}^{N^{\prime}} \rightarrow \mathbb{R}^{N}$, defined by

$$
P(w)=P^{\sigma}(w)=M^{\sigma} w^{\sigma}
$$

for each $w \in \mathcal{W}^{N^{\prime}}$, and $\sigma \in \Sigma_{E_{N^{\prime}}}$ such that $w \in K^{\sigma}$. 
Example 2 Consider again the mcst situation in Example 1. Then the contribution matrix is

$$
M^{\sigma}=\left(\begin{array}{cccccc}
\frac{1}{2} & \frac{1}{6} & 0 & \frac{1}{3} & 0 & 0 \\
0 & \frac{2}{3} & 0 & \frac{1}{3} & 0 & 0 \\
\frac{1}{2} & \frac{1}{6} & 0 & \frac{1}{3} & 0 & 0
\end{array}\right)
$$

and $w^{\sigma}=(10,18,20,24,24,26)^{t}$. Therefore $P(w)=M^{\sigma} w^{\sigma}=(16,20,16)^{t}$.

An alternative way of calculating $P(w)$, which will be useful in the following, is as nonnegative linear combination of $P\left(e^{\sigma, k}\right), k \in\left\{1, \ldots,\left|E_{N^{\prime}}\right|\right\}$, where $\sigma \in \Sigma_{E_{N^{\prime}}}$ is such that $w \in K^{\sigma}$ (see equation (2)). In formula,

$$
P(w)=w(\sigma(1)) P\left(e^{\sigma, 1}\right)+\sum_{k=2}^{\left|E_{N^{\prime}}\right|}(w(\sigma(k))-w(\sigma(k-1))) P\left(e^{\sigma, k}\right) .
$$

Note that since for each $\sigma \in \Sigma_{E_{N^{\prime}}}$ the connection vector $b^{\sigma,\left|E_{N^{\prime}}\right|}$ is the zero vector, the $P$-value of each mest situation $e^{\sigma, k} \in K^{\sigma}, k \in\left\{1, \ldots,\left|E_{N^{\prime}}\right|\right\}$, equals the connection vector corresponding to the graph $\left\langle N^{\prime}, F^{\sigma, k-1}\right\rangle$

$$
P\left(e^{\sigma, k}\right)=M^{\sigma} e^{\sigma, k}=\sum_{r=k}^{\left|E_{N^{\prime}}\right|}\left(b^{\sigma, r-1}-b^{\sigma, r}\right)=b^{\sigma, k-1} .
$$

Remark 2 It turns out that the $P$-value coincides with the Equal Remaining Obligations (ERO) rule. The ERO-rule has been introduced in Feltkamp et al. (1994) via an extension of Kruskal's algorithm. According to the ERO-rule, at each stage $k \in\left\{0,1, \ldots,\left|E_{N^{\prime}}\right|\right\}$ of the algorithm, each player $i \in N$ pays exactly the difference $f_{i}^{k}$ between remaining obligations $o_{i}^{k-1}$ and $o_{i}^{k}$, i.e. $f_{i}^{k}=o_{i}^{k-1}-o_{i}^{k}$ for each $i \in N$, where, as shown in Theorem 4.3 of Feltkamp et al. (1994), $o_{i}^{k}$ is equal to $b_{i}^{\sigma, k}$, with $\sigma$ such that $w \in K^{\sigma}$, as calculated in relation (3). An axiomatic characterization of the ERO-rule using the properties of NE (Non-Emptiness), FSC (Free-for-Source-Component), LOC (Local), Eff (Efficiency), ET (Equal Treatment) and IPCons (Inversely Proportional Consistency) is given there.

In the next section we provide an alternative axiomatic characterization.

\section{An axiomatic characterization of the $P$-value}

We call a map $F: \mathcal{W}^{N^{\prime}} \rightarrow \mathbb{R}^{N}$ assigning to every mest situation $w$ a unique cost allocation in $\mathbb{R}^{N}$ a solution. Some interesting properties for solutions of mest situations are the following. 
Property 1 The solution $F$ is efficient (EFF) if for each $w \in \mathcal{W}^{N^{\prime}}$

$$
\sum_{i \in N} F_{i}(w)=w(\Gamma)
$$

where $\Gamma$ is a minimum cost spanning network on $N^{\prime}$.

Property 2 The solution $F$ has the Equal Treatment (ET) property if for each $w \in \mathcal{W}^{N^{\prime}}$ and for each $i, j \in N$ with $C_{i}(w)=C_{j}(w)$

$$
F_{i}(w)=F_{j}(w)
$$

Property 3 The solution $F$ has the Upper Bounded Contribution (UBC) property if for each $w \in \mathcal{W}^{N^{\prime}}$ and every $\left(w, N^{\prime}\right)$-component $C \neq\{0\}$

$$
\sum_{i \in C \backslash\{0\}} F_{i}(w) \leq \min _{i \in C \backslash\{0\}} w(\{i, 0\}) .
$$

Property 4 The solution $F$ has the Cone-wise Positive Linearity (CPL) property if for each $\sigma \in \Sigma_{E_{N^{\prime}}}$, for each pair of mcst situations $w, \widehat{w} \in K^{\sigma}$ and for each pair $\alpha, \widehat{\alpha} \geq 0$, we have

$$
F(\alpha w+\widehat{\alpha} \widehat{w})=\alpha F(w)+\widehat{\alpha} F(\widehat{w}) .
$$

Proposition 2 The P-value satisfies the properties EFF, ET, UBC and CPL.

Proof Let $w \in \mathcal{W}^{N^{\prime}}$ and let $\sigma \in \Sigma_{E_{N^{\prime}}}$ be such that $w \in K^{\sigma}$. Then the following considerations hold:

i) Let $\sigma\left(t_{1}\right), \sigma\left(t_{2}\right), \ldots, \sigma\left(t_{n}\right)$, be the $n$ edges of the mcst $\Gamma$ corresponding to Kruskal order $\sigma$. These edges correspond to non-zero columns in $M^{\sigma}$ and then the sum of coordinates of each column equals 1 . Hence,

$$
\begin{gathered}
P(w)=M^{\sigma} w^{\sigma}=\sum_{r=1}^{n} w\left(\sigma\left(t_{r}\right)\right) M^{\sigma} e^{\sigma\left(t_{r}\right)} \\
\sum_{i \in N} P_{i}(w)=\sum_{r=1}^{n} w\left(\sigma\left(t_{r}\right)\right) \sum_{i \in N}\left(M^{\sigma} e^{\sigma\left(t_{r}\right)}\right)_{i}=\sum_{r=1}^{n} w\left(\sigma\left(t_{r}\right)\right)=w(\Gamma),
\end{gathered}
$$

which proves the EFF property. 
ii) Note that if $w$ is the zero function then the ET property is trivially satisfied. Consider $w \neq 0$ and define $k=\min \{j \mid w(\sigma(j))>0\}$. Then $w^{\sigma}$ is of the form $\left(0, \ldots, 0, w(\sigma(k)), \ldots, w\left(\sigma\left(\left|E_{N^{\prime}}\right|\right)\right)\right)^{t}$. Then for each $i \in N$

$$
P_{i}(w)=\left(M^{\sigma} w^{\sigma}\right)_{i}=\sum_{r=k}^{\left|E_{N^{\prime}}\right|}\left(b_{i}^{\sigma, r-1}-b_{i}^{\sigma, r}\right) w(\sigma(r)) .
$$

Let $C$ be a $\left(w, N^{\prime}\right)$-component and consider two users $i, j \in C$. By Remark 1 this means that $i$ and $j$ are connected in the graph $\left\langle N^{\prime}, F^{\sigma, k-1}\right\rangle$ and so also in $<N^{\prime}, F^{\sigma, r}>$ for every $r \in\left\{k, \ldots,\left|E_{N^{\prime}}\right|\right\}$. Then for each $r \in\left\{k, \ldots,\left|E_{N^{\prime}}\right|\right\}$

$$
b_{i}^{\sigma, r-1}-b_{i}^{\sigma, r}=b_{j}^{\sigma, r-1}-b_{j}^{\sigma, r} .
$$

Hence, by $(10), P_{i}(w)=P_{j}(w)$, which proves the ET property.

iii) If $w$ is the zero function then the UBC property is trivially satisfied. Consider $w \neq 0$ and let $C \neq\{0\}$ be a $\left(w, N^{\prime}\right)$-component. Note that there exists $m \in\left\{1, \ldots,\left|E_{N^{\prime}}\right|\right\}$ such that $\sigma(m) \subseteq C \cup\{0\}$ and $w(\sigma(m))=\min _{i \in C \backslash\{0\}} w(\{i, 0\})$. Define $k=\min \{j \mid w(\sigma(j))>0\}$. If $m<k$, then $0 \in C$ and we are done since nodes in $C \backslash\{0\}$ pay nothing according to $P(w)$. Instead, if $m \geq k$ then

$$
\begin{aligned}
& \sum_{i \in C \backslash\{0\}} P_{i}(w)= \\
& =\sum_{i \in C \backslash\{0\}} \sum_{r=k}^{m} w(\sigma(r))\left(b_{i}^{\sigma, r-1}-b_{i}^{\sigma, r}\right) \leq \\
& \leq w(\sigma(m)) \sum_{i \in C \backslash\{0\}} \sum_{r=k}^{m}\left(b_{i}^{\sigma, r-1}-b_{i}^{\sigma, r}\right)= \\
& =w(\sigma(m)) \sum_{i \in C \backslash\{0\}} b_{i}^{\sigma, k-1}= \\
& =w(\sigma(m))
\end{aligned}
$$

where in the first equality we use that $b_{i}^{\sigma, u}=0$ for all $u \in\left\{m, \ldots,\left|E_{N^{\prime}}\right|\right\}$ and for each $i \in C$, and in the last one we use the fact that all nodes in $C \backslash\{0\}$ are connected in the graph $\left\langle N^{\prime}, F^{\sigma, k-1}\right\rangle$. Note that (11) proves the UBC property.

iv) The CPL property follows trivially from the definition of $P$.

Theorem 1 The P-value is the unique solution which satisfies the properties $E F F, E T, U B C$ and $C P L$ on the class $\mathcal{W}^{N^{\prime}}$ of mcst situations.

Proof We already know by Proposition 2 that the $P$-value satisfies the four properties EFF, ET, UBC and CPL. To prove the uniqueness consider a map $\psi: \mathcal{W}^{N^{\prime}} \rightarrow \mathbb{R}^{N}$ satisfying EFF, ET, UBC and CPL. 
Let $\sigma \in \Sigma_{E_{N^{\prime}}}$ and $k \in\left\{1, \ldots,\left|E_{N^{\prime}}\right|\right\}$. First we will show that for each mcst situation $e^{\sigma, k} \in K^{\sigma}, \psi\left(e^{\sigma, k}\right)=P\left(e^{\sigma, k}\right)$. By UBC, for each $\left(e^{\sigma, k}, N^{\prime}\right)$ component $C \neq\{0\}$

$$
\sum_{i \in C \backslash\{0\}} \psi_{i}\left(e^{\sigma, k}\right) \leq \min _{i \in C \backslash\{0\}} w(\{i, 0\})= \begin{cases}0 & \text { if } 0 \in C \\ 1 & \text { if } 0 \notin C\end{cases}
$$

implying that

$$
\sum_{i \in N} \psi_{i}\left(e^{\sigma, k}\right)=\sum_{C \in \mathcal{C}\left(e^{\sigma, k}\right)} \sum_{j \in C \backslash\{0\}} \psi_{j}\left(e^{\sigma, k}\right) \leq\left|\mathcal{C}\left(e^{\sigma, k}\right)\right|-1=e^{\sigma, k}(\Gamma),
$$

where $\Gamma$ is a minimum spanning network on $N^{\prime}$ for mcst situation $e^{\sigma, k}$. By $\mathrm{EFF}$, we have $\sum_{i \in N} \psi_{i}\left(e^{\sigma, k}\right)=e^{\sigma, k}(\Gamma)$, and then inequalities in relation (12) are equalities. Finally, by ET, we find that for each $i \in N$

$$
\begin{aligned}
\psi_{i}\left(e^{\sigma, k}\right) & =\left\{\begin{array}{ll}
0 & \text { if } 0 \in C_{i}\left(e^{\sigma, k}\right) \\
\frac{1}{\left|C_{i}\left(e^{\sigma, k}\right)\right|} & \text { if } 0 \notin C_{i}\left(e^{\sigma, k}\right)
\end{array}=\left\{\begin{array}{ll}
0 & \text { if } 0 \in C_{i}\left(e^{\sigma, k}\right) \\
\frac{1}{n_{i}\left(F^{\sigma, k-1}\right)} & \text { if } 0 \notin C_{i}\left(e^{\sigma, k}\right)
\end{array}=\right.\right. \\
& =P_{i}\left(e^{\sigma, k}\right) .
\end{aligned}
$$

Now we show that for any mcst situation $w \in \mathcal{W}^{N^{\prime}}, \psi(w)=P(w)$. Let $\sigma \in \Sigma_{E_{N^{\prime}}}$ be such that $w \in K^{\sigma}$. From the CPL property of $\psi$ and equation (2) it follows

$$
\psi(w)=w(\sigma(1)) \psi\left(e^{\sigma, 1}\right)+\sum_{k=2}^{\left|E_{N^{\prime}}\right|}(w(\sigma(k))-w(\sigma(k-1))) \psi\left(e^{\sigma, k}\right) .
$$

Further, from (8), (13) and (14) we obtain $\psi(w)=P(w)$.

To prove the logical independence of the four properties we need to consider some other solutions on $\mathcal{W}^{N^{\prime}}$ :

i) $\alpha P$, an $\alpha$ multiple of the solution concept $P$, with $\alpha \in[0,1)$;

ii) $\epsilon$, such that $\epsilon_{i}(w)=\frac{w(\Gamma)}{|N|}$ and $i \in N$, where $\Gamma$ is a minimum spanning network on $N^{\prime}$ for mest situation $w$;

iii) $P^{\tau}$ where $\tau \in \Sigma_{N}$, the set of bijections on $N$. To introduce this solution we follow the same plan used for the $P$-value. Analogously 
to Definition 3, for each $\sigma \in \Sigma_{E_{N^{\prime}}}$ we define $P^{\sigma, \tau}(w)=M^{\sigma, \tau} w^{\sigma}$ for each mest situation $w$ in the cone $K^{\sigma}$. Similarly to Definition 1 , here $M^{\sigma, \tau} e^{k}=b^{\sigma, \tau, k-1}-b^{\sigma, \tau, k}$ where, for each $k \in\left\{1, \ldots,\left|E_{N^{\prime}}\right|-1\right\}$ and $i \in N, b^{\sigma, \tau, k}$ is such that

$$
b_{i}^{\sigma, \tau, k}= \begin{cases}1 & \text { if } i=\arg \min _{j \in C_{i}\left(e^{\sigma, k+1}\right)} \tau(j) \text { and } 0 \notin C_{i}\left(e^{\sigma, k+1}\right) \\ 0 & \text { otherwise; }\end{cases}
$$

in addition $b_{i}^{\sigma, \tau, 0}=1$ and $b_{i}^{\sigma, \tau,\left|E_{N^{\prime}}\right|}=0$ for each $i \in N$.

A variant of Proposition 1 holds also for the maps $P^{\sigma, \tau}, \sigma \in \Sigma_{E_{N^{\prime}}}$, and so this enables us to define the solution $P^{\tau}(w)=P^{\sigma, \tau}(w)=M^{\sigma, \tau} w^{\sigma}$ for each $w \in \mathcal{W}^{N^{\prime}}$, where $\sigma \in \Sigma_{E_{N^{\prime}}}$ is such that $w \in K^{\sigma}$. The solution $P^{\tau}$ turns out to coincide with the allocation $x_{N}$ introduced in Norde et al. (2001) via an algorithmic procedure (called the Subtraction Algorithm) for the computation of a population monotonic allocation scheme (pmas) of a most game.

iv) $\mathcal{D}$, such that $\left(w, N^{\prime}\right)$-components "pay" proportionally to their "distance" from the source, i.e. such that for each $i \in N$

$$
\mathcal{D}_{i}(w)= \begin{cases}\frac{1}{\left|C_{i}(w)\right|} \frac{\min _{j \in C_{i}(w)} w(\{j, 0\})}{\sum_{C \in \mathcal{C}(w)} \min _{j \in C \backslash\{0\}} w(\{j, 0\})} w(\Gamma) & \text { if } 0 \notin C_{i}(w) \\ 0 & \text { if } 0 \in C_{i}(w),\end{cases}
$$

where $\Gamma$ is a minimum spanning network on $N^{\prime}$ for mest situation $w$.

Proposition 3 The axioms EFF, ET, UBC and CPL are logically independent.

Proof The logical independence of the four properties follows from the following table.

\begin{tabular}{c|c|c|c|c} 
& EFF & ET & UBC & CPL \\
\hline \hline$\alpha P$ & no & yes & yes & yes \\
\hline$P^{\tau}$ & yes & no & yes & yes \\
\hline$\epsilon$ & yes & yes & no & yes \\
\hline $\mathcal{D}$ & yes & yes & yes & no \\
\hline
\end{tabular}

In order to prove the first row, note that by Proposition 2, also $\alpha P$ satisfies axioms ET, UBC and CPL but not EFF for each $\alpha \in \mathbb{R}^{+}$such that $\alpha<1$. 
By definition, $P^{\tau}$ satisfies the CPL property. Similarly, via a variant of the arguments used in Proposition 2, it is possible to prove that $P^{\tau}$ satisfies also EFF and UBC. In order to show that $P^{\tau}$ does not satisfy the ET property, consider the mcst situation $\left\langle N^{\prime}, w>\right.$ with $N^{\prime}=\{0,1,2\}$ and $w$ as depicted in Figure 2. Then $P^{\tau}(w)=(1,2)$ for $\tau=(2,1)$.

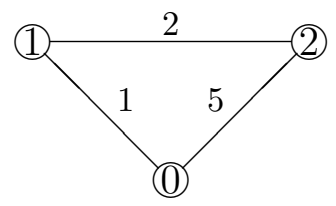

Figure 2: The mcst situation $<\{0,1,2\}, w>$.

To prove the third row, it is easy to see that $\epsilon$ satisfies EFF, ET and CPL. To see that $\epsilon$ does not satisfy the UBC property, consider again the mcst situation $\left\langle N^{\prime}, w\right\rangle$ with $N^{\prime}=\{0,1,2\}$ and $w$ as depicted in Figure 2. Then $\epsilon(w)=\left(\frac{3}{2}, \frac{3}{2}\right)$, i.e. player 1 in the $\left(w, N^{\prime}\right)$-component $C=\{1\}$ pays more than $\min _{i \in C \backslash\{0\}} w(\{i, 0\})=1$.

For the proof of the last row, note that $\mathcal{D}$ trivially satisfies EFF and ET. Let $w \in \mathcal{W}^{N^{\prime}}$ and let $\sigma \in \Sigma_{E_{N^{\prime}}}$ be such that $w \in K^{\sigma}$. The UBC property follows from the fact that for each component $C^{*} \in \mathcal{C}(w)$, if $0 \notin C^{*}$ then

$$
\begin{aligned}
& \min _{j \in C^{*}} w(\{j, 0\})=\frac{\min _{j \in C^{*} w(\{j, 0\})}}{\sum_{C \in \mathcal{C}(w)} \min _{j \in C \backslash\{0\}} w(\{j, 0\})} \sum_{C \in \mathcal{C}(w)} \min _{j \in C \backslash\{0\}} w(\{j, 0\}) \geq \\
& \geq \frac{\min _{j \in C^{*}} w(\{j, 0\})}{\sum_{C \in \mathcal{C}(w)} \min _{j \in C \backslash\{0\}} w(\{j, 0\})} w(\Gamma)=\sum_{j \in C^{*}} \mathcal{D}_{j}(w) .
\end{aligned}
$$

In order to prove that $\mathcal{D}$ does not satisfy the CPL property, consider the two mest situations $\left\langle N^{\prime}, w^{\prime}\right\rangle$ and $\left\langle N^{\prime}, w^{\prime \prime}>\right.$, with $N^{\prime}=\{0,1,2\}$ and $w^{\prime}$, $w^{\prime \prime}$ as depicted in Figure 3.
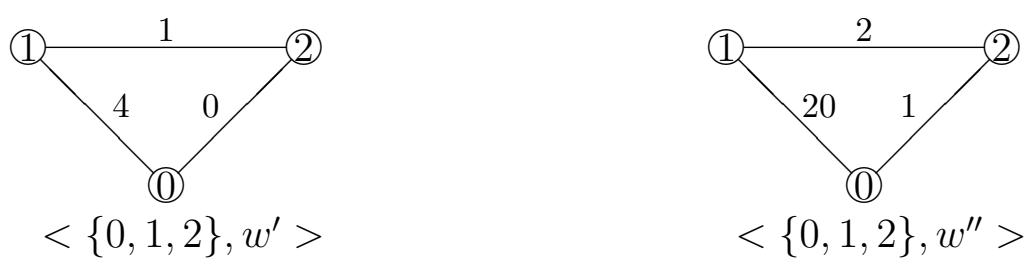

Figure 3: Two mcst situations in the same Kruskal cone.

Note that $w^{\prime}, w^{\prime \prime} \in K^{\sigma}$ with $\sigma(1)=\{0,2\}, \sigma(2)=\{1,2\}$ and $\sigma(3)=\{0,1\}$. Then $\mathcal{D}\left(w^{\prime}\right)=(1,0)$ and $\mathcal{D}\left(w^{\prime \prime}\right)=\left(\frac{60}{21}, \frac{3}{21}\right)$.

Differently, the sum of the two mest situations $w^{\prime}+w^{\prime \prime}$ is the most situation $<N^{\prime}, w^{\prime}+w^{\prime \prime}>$ with $w^{\prime}+w^{\prime \prime}$ depicted in Figure 4. Finally, $\mathcal{D}\left(w^{\prime}+w^{\prime \prime}\right)=\left(\frac{96}{25}, \frac{4}{25}\right) \neq\left(\frac{81}{21}, \frac{3}{21}\right)=\mathcal{D}\left(w^{\prime}\right)+\mathcal{D}\left(w^{\prime \prime}\right)$. 


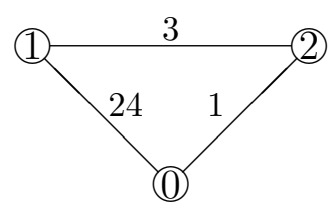

Figure 4: The mcst situation $<\{0,1,2\}, w^{\prime}+w^{\prime \prime}>$.

\section{Concluding remarks}

In this paper a solution for mcst situations, the $P$-value, has been introduced. Also, an axiomatic characterization of the $P$-value using the properties EFF (Efficiency), ET (Equal Treatment), UBC (Upper Bounded Contribution) and CPL (Cone-wise Positive Linearity) is given.

One can prove that

$$
P(w)=\frac{1}{n !} \sum_{\tau \in \Sigma_{N}} P^{\tau}(w)
$$

where $w \in \mathcal{W}^{N^{\prime}}$ and $P^{\tau}(w)$ are as described in Section 4. It is shown in Norde et al. (2001) that $\left[P_{i}^{\tau}\left(w_{\mid S \cup\{0\}}\right)\right]_{S \in 2^{N} \backslash\{\emptyset\}, i \in S}$ is a pmas.

The $P$-value for mcst situations induces a cost sharing rule for mcst games, which we call the $\Pi$-value (Potters value). The $\Pi$-value is the map $\Pi: \mathcal{M C S T}^{N} \rightarrow \mathbb{R}_{+}^{N}$ obtained by $\Pi\left(c_{w}\right)=P(w)$, where $w \in \mathcal{W}^{N^{\prime}}$. It follows that $\Pi$ is positive linear on the cone $\mathcal{G}^{\sigma}$, i.e. for all $c_{w}, c_{w^{\prime}} \in \mathcal{G}^{\sigma}$ and all $\alpha, \alpha^{\prime} \in \mathbb{R}_{+}$it holds

$$
\Pi\left(\alpha c_{w}+\alpha^{\prime} c_{w^{\prime}}\right)=\alpha \Pi\left(c_{w}\right)+\alpha^{\prime} \Pi\left(c_{w^{\prime}}\right) .
$$

Moreover, (15) implies that the $\Pi$-value is a population monotonic allocation rule. To be more concrete, let us denote by $c_{w}^{S}$ the subgame of $c_{w}$ with player set $S, S \subseteq N$, defined by $c_{w}^{S}(T)=c_{w}(T)$, for each $T \subseteq S$. The $\Pi$-value assigns to each $c_{w}^{S} \in \mathcal{M C S} \mathcal{T}^{S}$ the $P$-value of the mcst subsituation $w_{\mid S^{\prime}}$, where $S^{\prime}=S \cup\{0\}$. In formula $\Pi\left(c_{w}^{S}\right):=P\left(w_{\mid S^{\prime}}\right)$ for each $c_{w}^{S} \in \mathcal{M C S T}^{S}$. Then, $\left[\Pi_{i}\left(c_{w}^{S}\right)\right]_{S \in 2^{N} \backslash\{\emptyset\}, i \in S}$ is a pmas.

In Tijs et al. (2003) we focus on other monotonicity properties of the $P$-value like cost monotonicity and drop-out monotonicity.

\section{References}

Aarts, H. (1994), Minimum cost spanning tree games and set games, PhD Dissertation, University of Twente, The Netherlands. 
Bird, C.G. (1976), On cost allocation for a spanning tree: A game theoretic approach, Networks, 6, 335-350.

Dutta, B., Kar, A. (2002), Cost monotonicity, consistency and minimum cost spanning tree games, University of Warwick, Mimeo.

Feltkamp, V. (1995), Cooperation in controlled network structures, PhD Dissertation, Tilburg University, The Netherlands.

Feltkamp, V., Tijs, S., Muto, S. (1994), On the irreducible core and the equal remaining obligations rule of minimum cost spanning extension problems, CentER DP 106, Tilburg University, The Netherlands.

Granot, D., Huberman, G. (1981), On minimum cost spanning tree games, Mathematical Programming, 21, 1-18.

Kar, A. (2002), Axiomatization of the Shapley value on minimum cost spanning tree games, Games and Economic Behavior, 38, 265-277.

Kent, K.J., Skorin-Kapov, D. (1996), Population monotonic cost allocations on MSTs, DP, State University of New York at Stony Brook.

Kruskal, J.B. (1956), On the shortest spanning subtree of a graph and the traveling salesman problem, Proceedings of the American Mathematical Society, $7,48-50$.

Kuipers, J. (1993), On the core of information graph games, International Journal of Game Theory, 21, 339-350.

Moretti, S., Norde, H., Pham Do, K.H., Tijs, S. (2002), Connection problems in mountains and monotonic allocation schemes, Top, 10, 83-99.

Norde, H., Moretti, S., Tijs, S. (2001), Minimum cost spanning tree games and population monotonic allocation schemes, CentER DP 2001-18, Tilburg University, The Netherlands (to appear in European Journal of Operational Research).

Sprumont, Y. (1990), Population monotonic allocation schemes for cooperative games with transferable utility, Games and Economic Behavior, 2, 378-394. 
Tijs, S., Moretti, S., Norde, H., Branzei, R. (2003), A class of cost monotonic sharing rules for minimum cost spanning tree situations, Working paper. 